\title{
Pathogens and commensals fight it out
}

Our gut is colonized by trillions of commensal bacteria, which have many physiological roles, for example in nutrition and immunity. So how do pathogenic enteric bacteria, such as enterohaemorrhagic Escherichia coli (EHEC) and enteropathogenic E. coli (EPEC), manage to disrupt this balance and colonize the host? Using a mouse model, Núñez and colleagues reveal that the ability of enteric pathogens to outcompete the gut microbiota is influenced by their expression of virulence genes and by competition for nutrients.

To examine the interaction between enteric pathogens and the gut microbiota, the authors infected germ-free mice (which lack microorganisms) and specific pathogen-free (SPF) mice (which lack specific pathogenic microorganisms) with the mouse pathogen Citrobacter rodentium, which, like EHEC and EPEC, colonizes the host by forming attaching and effacing (A-E) lesions. Germ-free mice carried tenfold more $C$. rodentium than SPF mice and, unlike SPF mice, could not clear the bacteria despite mounting comparable immune responses.

Colonization of the host by A-E pathogens is known to depend on virulence factors encoded by the locus of enterocyte effacement (LEE), expression of which is induced by LEE-encoded regulator (Ler). However, Ler-deficient $C$. rodentium could still colonize germ-free mice, which suggests that LEE-mediated virulence is not required for colonization in the absence of the gut microbiota. Importantly, the authors observed that expression of LEE-encoded virulence genes regulated bacterial localization at different stages of infection. At day 21 post-infection, wild-type C. rodentium relocalized from the gut epithelial surface (which normally lacks commensal bacteria) to the intestinal lumen of germ-free mice. By contrast, Ler-deficient bacteria were not observed on the intestinal

surface but were localized to the lumen when assessed at day 5 post-infection. As Ler-regulated genes in
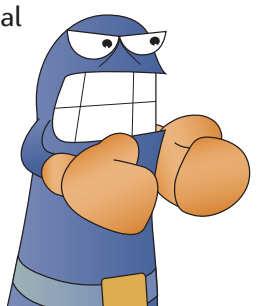

wild-type bacteria were downregulated 12 days after infection, the authors conclude that C. rodentium relocalization is mediated by downregulation of LEE-encoded virulence genes at late stages of infection.

After relocalizing to the intestinal lumen, C. rodentium would have to compete with the resident commensal bacteria. To determine whether different commensals have distinct abilities to outcompete $C$. rodentium, the authors infected germ-free mice with the pathogen and colonized them with either $E$. coli or two Bacteroides species 21 days later. Inoculation with E. coli, but not Bacteroides thetaiotaomicron or Bacteroides vulgatus, led to a significant decrease in $C$. rodentium burden. Further analysis demonstrated that both $E$. coli and $C$. rodentium showed optimal growth on monosaccharides, whereas $B$. thetaiotaomicron and $B$. vulgatus could also grow on polysaccharides, suggesting that E. coli and $C$. rodentium compete for carbohydrates.

To test this hypothesis in vivo, the authors colonized germ-free mice with $B$. thetaiotaomicron 21 days after infection with $C$. rodentium and then fed the mice either a simple diet of monosaccharides or a conventional diet containing monosaccharides and polysaccharides. C. rodentium burden rapidly decreased in mice fed the simple diet, whereas no effect was observed in mice fed the conventional diet.

These findings reveal that colonization of the gut by pathogenic bacteria is regulated by their virulence genes and, perhaps as expected, also depends on their ability to compete with commensal bacteria that are metabolically similar. The authors suggest that altering the balance of the resident microbiota towards bacteria that can directly compete with a pathogen would be a viable therapeutic strategy.

Rachel David

ORIGINAL RESEARCH PAPER Kamada, N. et al. Regulated virulence controls the ability of a pathogen to compete with the gut microbiota. Science 10 May 2012 (doi:10.1126/science.1222195)

colonization

of the gut by

pathogenic

bacteria is

regulated by

their virulence

genes

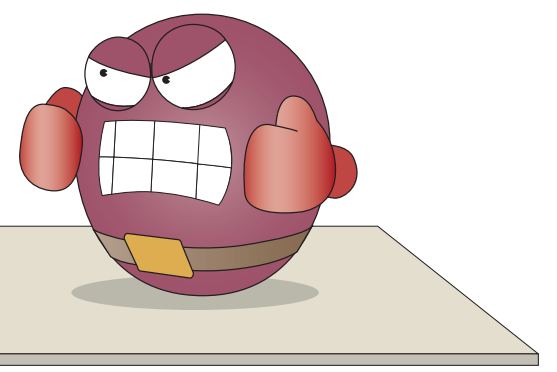

